\title{
Battle of COVID-19
}

\author{
Gurmeet Kaur
}

\begin{abstract}
It was in January 2021 the free vaccination against COVID-19 commenced in India and the government urged all of its citizens to get vaccinated which was expected to be the largest vaccination program in the world. Out of the various COVID-19 vaccines that were under various stages of clinical trials in India, four were developed in the country. Covishield (the name employed in India for the Oxford-AstraZeneca vaccine) and Covaxin, the home-manufactured vaccine produced by Bharat Biotech had received approval from the Drug Controller of India. Indian manufacturers had stated that they can meet the country's future needs for COVID-19 vaccines. The manpower and cold-chain infrastructure established before the pandemic was sufficient for the initial vaccination of 30 million healthcare workers and was strengthened further to cater to the entire population subsequently. The Indian government had taken urgent measures to expand the country's vaccine manufacturing capacity and has also developed an efficient digital system to address and monitor all the aspects of vaccine administration. It brings in the sequential events in the battle against COVID-19 in India.
\end{abstract}

Keywords: Covaxin, COVID vaccination, COVID-19, Covishield.

Journal of Medical Academics (2021): 10.5005/jp-journals-10070-0071

It was the first case of novel coronavirus infection detected in the Wuhan province of China which threw light on the world of microbes and their pandemic potential. Globally, the efforts were focused on breaking the chain of transmission. ${ }^{1-6}$ Shortly, it was understood that the herd immunity of COVID-19 could be a breakthrough, thereby introducing the urgent need for effective COVID-19 vaccines. ${ }^{7}$ Hence, the vaccine development has commenced the accomplishment of prevention of COVID-19 disease though the emerging mutant variants continue to pose a threat to the world. ${ }^{8}$

India with its population of 1,380 million has taken a huge leap among the countries with the highest vaccination coverage despite all the logistics and hesitancy hurdles. India's robust vaccine development program crafted the domestic manufacture of the COVID-19 vaccine which not only sufficed the needs of the citizens of India but also provided the vaccine distribution to all the countries that could not afford the lucrative and luxurious vaccines of the Western world. In India, the data derived from completed clinical trials and follow-up studies of various vaccines supported their eligibility for emergency authorization, quality control through the efficient cold chain, enormous production, and cost regulation of these vaccines made them inexpensive to even the underprivileged nations of the world. ${ }^{9,10}$

\section{Covid-19 Vaccine Candidates in India}

COVID-19 vaccine candidates in India are among the prominent vaccine manufacturers in the world. Apart from India's indigenous COVID-19 vaccines, local pharmaceutical and biotech companies signed joint agreements with foreign-based vaccine contractors which ranged from steering clinical trials to large-scale manufacturing of vaccines and their distribution. ${ }^{11}$

\section{Covishield by the Serum Institute of India}

Serum Institute of India (SII), Pune, had signed agreements with manufacturers like Oxford-AstraZeneca, Codagenix, and Novavax. It is now producing at a large scale, the Oxford-AstraZeneca Adenovirus vector-based vaccine AZD1222 (which is named
Department of Community Medicine, Army College of Medical Sciences, Delhi, India

Corresponding Author: Gurmeet Kaur, Department of Community Medicine, Army College of Medical Sciences, Delhi, India, Phone: +91 9953203473, e-mail: drgurmeetkaurkumar@gmail.com

How to cite this article: Kaur G. Battle of COVID-19. J Med Acad 2021;4(1):1-4.

Source of support: Nil

Conflict of interest: None

"Covishield" in India), and has stored >50 million doses. ${ }^{12}$ The company continues to produce 100 million doses per month since January 2021. The ICMR supported the clinical trials of the Covishield vaccine developed with the principal stock from Oxford-AstraZeneca. The SII and ICMR have together directed a Phase II/III, observer-blind, randomized, controlled trial in healthy adults at 14 foci in India, for evaluation of the safety of Covishield (made in India) against the original Oxford-ChAdOx1 in the prevention of COVID-19 disease. One thousand and six hundred eligible participants of $\geq 18$ years of age or older enrolled in the study, 400 participants participated in immunogenicity cohort which were randomly distributed in a 3:1 ratio to obtain either Covishield or Oxford-ChAdOx1, respectively. The left 1,200 participants of the safety cohort were randomly distributed in a 3:1 ratio to obtain either Covishield or Placebo, respectively. The safety, immunogenicity, and efficacy data of clinical studies outside India depicted the vaccine efficacy to be $70.42 \%$ among 23,745 participants who were administered two doses of ChAdOx 1 containing $5 \times 10^{10}$ viral particles. ${ }^{13}$ The clinical trial in India was found to be analogous with the data from previous trials conducted outside of India. The SII clinical trials in India for Covovax (NVX-CoV2373) developed in partnership with Novavax are hopeful of launching their vaccine by end of 2021. The US-based pharma asserts that their COVID jab was $89.3 \%$ effective in a UK trial. ${ }^{14,15}$

(c) The Author(s). 2021 Open Access This article is distributed under the terms of the Creative Commons Attribution 4.0 International License (https://creativecommons. org/licenses/by-nc/4.0/), which permits unrestricted use, distribution, and non-commercial reproduction in any medium, provided you give appropriate credit to the original author(s) and the source, provide a link to the Creative Commons license, and indicate if changes were made. The Creative Commons Public Domain Dedication waiver (http://creativecommons.org/publicdomain/zero/1.0/) applies to the data made available in this article, unless otherwise stated. 


\section{Covaxin by Bharat Biotech Ltd}

Covaxin $^{\text {TM }}$, India's first domestic vaccine manufactured by Bharat Biotech International Limited, in collaboration with the National Institute of Virology of ICMR. It is an inactivated virus vaccine, developed in Vero cells by combining with Alhydroxiquim-II (Algel-IMDG), chemosorbed imidazoquinoline onto aluminum hydroxide gel, as an adjuvant to boost immune response, thereby providing long-lasting immunity. This technology is authorized under a licensing agreement with Kansas-based ViroVax. The use of the Imidazoquinoline class of adjuvants (TLR7/8 agonists), shifts the T-cell response toward Th1, a T-helper 1 phenotype (which is considered safer than Th2 responses against SARS-CoV-2) and reduces the risk of immunopathologically mediated enhanced disease. ${ }^{16}$ Bharat Biotech Ltd and ICMR conducted Phase-I and Phase-II clinical trials on 800 subjects and the results have confirmed that the vaccine is safe and provides a robust immune response and protection. The Phase-III efficacy trial was conducted on 25,800 volunteers who have been vaccinated across India, decreed the vaccine to be safe. The company produced 150 million doses by July-August 2021 and will be ready with 700 million doses by the end of 2021.

\section{ZyCoV-D by Cadila Healthcare (Zydus Cadila)}

ZyCoV-D by Cadila Healthcare, Ahmedabad, is yet another domestic vaccine, conceptualized on the new plasmid DNA vaccine technology, collaborated with the Department of Biotechnology, Government of India. Plasmid DNA technologybased vaccines are not yet licensed for public use. Plasmids are used as vectors to directly deliver the DNA encoding the target antigens into the body of the recipient. Sequence encoding for the pathogen's antigen is engineered into recombinant plasmid DNA. It is used as the vaccine vector so that the vaccine antigens are directly produced by human cells, thus eliciting an immune response. The Phase-I trials of this vaccine began on July 13, 2020, on volunteers of $18-55$ years of age. As ZyCoV-D showed promise in a Phase-I study, and the drugmaker Cadila is currently finishing Phase-II and III trials on over 1,000 volunteers across nine sites. It is the first DNA needle-free vaccine that has received emergency approval from NTAGI for vaccination of adolescents 12 years and above with comorbidities in 3 doses 1 month apart in October $2021 .^{16-18}$

\section{Sputnik V by Dr Reddy's Laboratories}

Gam-COVID-Vac, trade-named Sputnik V, is a COVID-19 vaccine developed by the Gamaleya National Center of Epidemiology and Microbiology of Moscow, Russia. Sputnik V is a two-vector viral vaccine based on human adenoviruses. Sputnik $V$ uses adenoviruses Ad5 and Ad26. ${ }^{19}$ The recombinant adenovirus types 26 and 5 are biotechnology-derived and contain the SARS-CoV-2 S protein cDNA. Both of them are administered into the deltoid muscle. The Ad26-based vaccine is used on the first day and the Ad5 vaccine is used on the 21st day to boost immune responses. Russia's Sputnik V vaccine stipulates storage at a temperature not higher than $-18^{\circ} \mathrm{C}$.

Dr Reddy's Laboratories, located in Hyderabad, have received regulatory approval from the DCGI to conduct mid-to-late-stage human trials for Russia's Sputnik V vaccine in India. Russia's RDIFGamaleya Institute has signed agreements with more than one Indian company for the large-scale manufacture of their Sputnik $\checkmark$ vaccine.

\section{Covid-19 Vaccination In India}

The government of India had constituted a National Expert Group on Vaccine Administration for COVID-19 (NEGVAC) to guide all aspects of COVID-19 vaccine administration in India. ${ }^{20}$ According to NEGVAC, the COVID-19 vaccine was offered first to healthcare workers, frontline workers, and to persons above 50 years of age (with first preference for those above 60), followed by persons younger than 50 years of age with associated comorbidities. The government had set up a committee comprising experts from various specialties including oncology, nephrology, pulmonology, and cardiology to define the clinical criteria, based on which people with comorbidities should be prioritized for COVID-19 vaccination. Committee has recommended that anyone with a congenital heart disease that leads to pulmonary arterial hypertension, end-stage kidney disease, or cancers such as lymphoma, leukemia, myeloma, decompensated liver cirrhosis, primary immune deficiency conditions, and sickle cell anemia were included in the priority. The latest electoral roll for the general election was used to identify the population aged 45 years or more. The cut-off date for determining the age was January 1, 2021. There was a provision for self-registration for vaccination, for those eligible persons who have been missed out from the rolls for one reason or other, after giving some proof of identity. After vaccinating nearly 300 million of the population in the first phase, the remaining population received the vaccine based on the disease epidemiology and vaccine availability.

The Government of India had arranged to procure 600 million doses of the COVID-19 vaccine from the manufacturers highlighted above and is negotiating for another billion doses. Covishield, produced by SIl, and Covaxin produced by Bharat Biotech Ltd were procured by the government, and are administered initially. Nevertheless, the government may alter its strategy, as and when the other vaccines are cleared for administration after the clinical trials. While obtaining the vaccine was the first requirement, distribution, and vaccination of the huge Indian population presents a significant logistic challenge that was overcome by the support of the Government.

The companies SII, Bharat Biotech, and Pfizer India had applied for "emergency use authorization" of their vaccines. All their applications were reviewed by the expert panel at the Central Drugs Standard Control Organization (CDSCO) for their suitability for vaccination in this country. During the second round of discussions, Covishield, the vaccine candidate from Pune-based SII, was approved for emergency use by the Subject Expert Committee (SEC) of DCGI on January 1, 2021. They had approved the vaccine to be given in two doses 4 to 12 weeks apart. This time interval is similar to that employed by the UK, and the company is allowed to deploy its vaccines to priority groups, even though a full safety assessment has not been completed. Bharat Biotech was asked to furnish more data demonstrating the efficacy of its candidate, Covaxin. On January 2, 2021, the SEC gave its approval to Bharat Biotech's Covaxin coronavirus vaccine also for emergency use. These recommendations, along with rollout modalities, were taken up by the DCGI.

It was established that Covaxin's ability to protect against mutant strains of the novel coronavirus, detected in the UK and 30 other countries. The approval of the SEC of DCGI only means that the firm will no longer require to have a placebo group in its ongoing clinical trial, and will vaccinate people in an open-label format. The safety and efficacy of the drug were to be closely monitored. 
However, Bharat Biotech announced on March 3, 2021, the results of the third round of clinical trials showed that Covaxin was $80.7 \%$ effective in preventing COVID-19. After going through Covaxin's Phase 3 trial data, the subject expert committee gave emergency use authorization for this vaccine. People above 50 years of age and above with comorbidities had been prioritized for vaccination by the government, a decision on the administration of Covishield and Covaxin was given in March 2021. It was in June 2021 both vaccines were open for public use above 18 years of age. The use of the vaccine in prioritized populations, along with the continued implementation of other public health measures and community participation led to reducing the impact of COVID-19.

\section{Program Implementation}

COVID-19 vaccination was totally under government control. Highlevel coordination at national, state, and district levels had been established for effective cooperation and collaboration among the key departments involved in COVID-19 vaccination. Twenty-three ministries/departments and numerous developmental partners were involved in planning for the COVID-19 vaccine introduction. Their roles had been described in the operational guidelines issued by the Ministry of Health and Family Welfare, Government of India. ${ }^{20}$ Co-WIN system was linked to existing UIP programs and it meticulously monitor and follow-up on the immunized individuals. The Co-WIN system was used not only to track enlisted beneficiaries but also to ensure that only pre-registered beneficiaries were vaccinated in accordance with the prioritization. Enlisted beneficiaries selected vaccination sites nearest to their home. Autogenerated SMS/email intimations were sent to the beneficiaries, vaccinators, mobilizers, and supervisors about the date, time, and place of the session. To observe the staggered approach, beneficiaries were advised by mobilizers to come to the session as per the staggered time slot to prevent over-crowding at the session site. ${ }^{21}$ As per the guidelines issued by the Centre for the COVID-19 inoculation drive, 100 people were injected in each session per day. People were monitored for 30 minutes after administering the shots for any adverse event. Based on the initial experience, some vaccination sites had been permitted to work for 24 hours every day and the number of people to be injected in each session had been increased up to 200 , to speed up the vaccination. Experience gained in the vaccination of the first round was helpful for the improvement of the second and subsequent cycles. ${ }^{22}$

\section{Conclusion}

The purpose of this perspective was to highlight the overall crux of the vaccine development and vaccination strategies that were implemented during a pandemic in a densely populated country (India). It presents a baseline for future pandemic preparedness and effectively tailors and refine the strategies that will help the population at large. ${ }^{23}$

India is in a privileged position in producing affordable medical, surgical, and essential generic medicines for the world. It is also well-known that India is the world's largest manufacturer and worldwide distributor of vaccines. The current COVID-19 pandemic has triggered rapid development, emergency use authorization, and unprecedented collaborative efforts from various stakeholders. Vaccination proved to be a cost-effective strategy for survival and a better quality of life for the people as well as for the revival of the economy of India. A periodic re-evaluation of the vaccine platforms, the efficacy and safety of COVID-19 vaccination through postmarketing surveillance is of paramount importance and requires long-term follow-up. This should account for both successes and failures, outstanding benefits, and/or its superiority over other types of pharmacological and nonpharmacological treatment regimens. Studies are needed nationally and globally, along with transparent sharing of data and reports among all participating companies, institutions, and nations. ${ }^{24}$ It is mandatory for periodic evaluation and re-strategizing COVID-19 management plans. These data analyzes can hold the keys to the future effective public health management of COVID-19. India's experience in immunization for COVID-19 offers tips for strategy preparation, not only for countries with similar economic strength and health facilities but also for the world at large.

\section{References}

1. Gupta R, Kumar VM, Tripathi M, et al. Guidelines of the Indian Society for Sleep Research (ISSR) for practice of sleep medicine during COVID19. Sleep Vigil 2020;4(2):1-12. DOI: 10.1007/s41782-020-00097-2.

2. Pandi-Perumal SR, Gulia KK, Gupta D, et al. Dealing with a pandemic: the Kerala model of containment strategy for COVID-19. Pathog Glob Health 2020;114(5):232-233. DOI: 10.1080/20477724.2020.1778392.

3. Gulia KK, Kumar VM. Reverse quarantine in Kerala: managing the 2019 novel coronavirus in a state with a relatively large elderly population. Psychogeriatrics 2020;20(5):794-795. DOI: 10.1111/psyg.12582.

4. Gulia KK, Kumar VM. Importance of sleep for health and wellbeing amidst COVID-19 pandemic. Sleep Vigil May 2020;4(1):1-2. DOI: 10.1007/s41782-020-00087-4.

5. Cardinali DP, Brown GM, Reiter RJ, et al. Elderly as a high-risk group during COVID-19 pandemic: effect of circadian misalignment, sleep dysregulation and melatonin administration. Sleep Vigil 2020;26(2):17. DOI: 10.1007/s41782-020-00111-7.

6. Gupta I, Baru R. Economics \& ethics of the COVID-19 vaccine: how prepared are we? Indian J Med Res 2020;152(1\&2):153-155. DOI: 10.4103/ijmr.IJMR_3581_20.

7. Vignesh R, Shankar EM, Vijayakumar V, et al. Is herd immunity against SAR-CoV2 a silver lining? Front Immunol 2020;11:586781. DOI: 10.3389/ fimmu.2020.586781.

8. Le TT, Cramer JP, Chen R, et al. Evolution of the COVID-19 vaccine development landscape. Nat Rev Drug Discov 2020;19(10):667-668. DOI: 10.1038/d41573-020-00151-8.

9. Kochhar S, Salmon DA. Planning for COVID-19 vaccines safety surveillance. Vaccine 2020;38(40):6194-6198. DOI: 10.1016/j. vaccine.2020.07.013.

10. Krause P, Fleming TR, Longini I, et al. COVID-19 vaccine trials should seek worthwhile efficacy. Lancet 2020;396(10253):741-743. DOI: 10.1016/S0140-6736(20)31821-3.

11. Zhang $\mathrm{Y}$, Zeng $\mathrm{G}$, Wang $\mathrm{H}$, et al. Safety tolerability, and immunogenicity of an inactivated SARS-CoV-2 vaccine in healthy adults aged 18-59 years: a randomised, double-blind, placebo-controlled, phase 1/2 clinical trial. Lancet Infect Dis 2020;21(1):39-51. DOI: 10.1016/S14733099(20)30843-4.

12. Bar-Zeev N, Kochhar S. Expecting the unexpected with COVID-19 vaccines. Lancet Infect Dis 2020;21(2):150-151. DOI: 10.1016/S14733099(20)30870-7.

13. Vaccine information, ICMR New Delhi-COVID-19 vaccine. https:// vaccine.icmr. org.in/covid-19-vaccine (2021).

14. Voysey M, Clemens SAC, Madhi SA, et al. Safety and efficacy of the ChAdOx1 nCoV-19 vaccine (AZD1222) against SARS-CoV-2: an interim analysis of four randomised controlled trials in Brazil, South Africa, and the UK. Lancet 2021;397(10269):99-111. DOI: 10.1016/S01406736(20)32661-1.

15. Keech C, Albert G, Cho I, et al. Phase 1-2 trial of a SARS-CoV-2 recombinant spike protein nanoparticle vaccine. $\mathrm{N}$ Engl J Med 2020;383(24):2320-2332. DOI: 10.1056/NEJMoa2026920. 
16. Ella R. A Phase 1: safety and immunogenicity trial of an inactivated SARS-CoV-2 vaccine BBV152, a double-blind, randomised, Phase 1 trial. Lancet Infect Dis 2021;21(7):950-961. DOI: 10.1016/S14733099(20)30942-7(2021)

17. Liu Y, Wang K, Massoud TF, et al. SARS-CoV-2 vaccine development:an overview and perspectives. ACS Pharm TransI Sci 2020;3(5):844-858. DOI: $10.1021 /$ acsptsci.0c00109.

18. Momin T, Kansagra $K$, Patel $H$, et al. Safety and immunogenicity of a DNA SARS-CoV-2 vaccine (ZyCoV-D): Results of an open-label, nonrandomized phase I part of phase I/II clinical study by intradermal route in healthy subjects in India. E Clin Med 2021;38:101020. DOI: 10.1016/j.eclinm.2021.101020.

19. Logunov DY, Dolzhikova IV, Zubkova OV, et al. Safety and immunogenicity of an rAd26 and rAd5 vector-based heterologous prime-boost COVID-19 vaccine in two formulations: two open, non-randomised phase 1/2 studies from Russia. Lancet 2020; 396(10255):887-897. DOI: 10.1016/S0140-6736(20)31866-3.
20. Ministry of Health and Family Welfare (2020) COVID-19 Vaccine Operational Guidelines. Ministry of Health and Family Welfare, Government of India. 28 December 2020. https://main.mohfw.gov. in/sites/default/files/COVID19VaccineOG111Chapter16.pdf. (Accessed on 04 June 2021).

21. Bhuyan A. India begins COVID-19 vaccination amid trial allegations. Lancet 2021;397(10271):P264. DOI: 10.1016/S0140-6736(21) 00145-8.

22. Sapkal GN, Yadav PD, Ella R, et al., Neutralization of UK-variant VUI-202012/01 with COVAXIN vaccinated human serum. Preprint at https://www.biorxiv.org/content/10.1101/2021.01.26.426986v2 2021.

23. Rego GNA, Nucci MP, Alves AH, et al. Current clinical trials protocols and the global effort for immunization against SARS-CoV-2. Vaccines 2020;8(3):474. DOI: 10.3390/vaccines8030474.

24. Wolemonwu VC. Human challenge trials for a COVID-19 vaccine: should we bother about exploitation? Voices Bioeth 2020;6:1-6. 